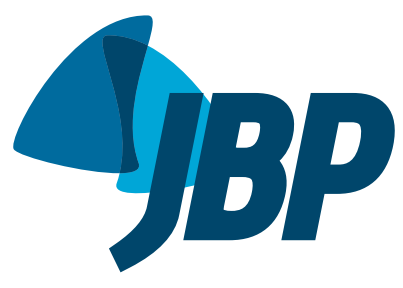

\title{
Correlation between the severity of critically ill patients and clinical predictors of bronchial aspiration
}

Gisele Chagas de Medeiros ${ }^{1}$, Fernanda Chiarion Sassi ${ }^{2}$, Lucas Santos Zambom³, Claudia Regina Furquim de Andrade ${ }^{2}$

1. Divisão de Fonoaudiologia, Instituto Central, Hospital das Clínicas, Faculdade de Medicina, Universidade de São Paulo, São Paulo (SP) Brasil.

2. Departamento de Fisioterapia, Fonoaudiologia e Terapia Ocupacional Faculdade de Medicina, Universidade de São Paulo, São Paulo (SP) Brasil.

3. Disciplina de Emergências Clínicas, Faculdade de Medicina, Universidade de São Paulo, São Paulo (SP) Brasil.

Submitted: 6 August 2015. Accepted: 27 January 2016.

Study carried out in the Divisão de Fonoaudiologia, Instituto Central, Hospital das Clínicas, Faculdade de Medicina, Universidade de São Paulo, São Paulo (SP) Brasil.

\begin{abstract}
Objective: To determine whether the severity of non-neurological critically ill patients correlates with clinical predictors of bronchial aspiration. Methods: We evaluated adults undergoing prolonged orotracheal intubation ( $>48 \mathrm{~h}$ ) and bedside swallowing assessment within the first $48 \mathrm{~h}$ after extubation. We collected data regarding the risk of bronchial aspiration performed by a speech-language pathologist, whereas data regarding the functional level of swallowing were collected with the American SpeechLanguage-Hearing Association National Outcome Measurement System (ASHA NOMS) scale and those regarding health status were collected with the Sequential Organ Failure Assessment (SOFA). Results: The study sample comprised 150 patients. For statistical analyses, the patients were grouped by ASHA NOMS score: ASHA1 (levels 1 and 2), ASHA2 (levels 3 to 5); and ASHA3 (levels 6 and 7). In comparison with the other patients, those in the ASHA3 group were significantly younger, remained intubated for fewer days, and less severe overall clinical health status (SOFA score). The clinical predictors of bronchial aspiration that best characterized the groups were abnormal cervical auscultation findings and cough after swallowing. None of the patients in the ASHA 3 group presented with either of those signs. Conclusions: Critically ill patients 55 years of age or older who undergo prolonged orotracheal intubation ( $\geq 6$ days), have a SOFA score $\geq 5$, have a Glasgow Coma Scale score $\leq 14$, and present with abnormal cervical auscultation findings or cough after swallowing should be prioritized for a full speech pathology assessment.
\end{abstract}

Keywords: Deglutition; Deglutition disorders; Intubation, intratracheal; Pneumonia, aspiration; Intensive care units.

\section{INTRODUCTION}

Dysphagia after prolonged orotracheal intubation (OTI)-longer than $48 \mathrm{~h}^{(1-3)}$-is defined as the inability to effectively transfer food from the mouth into the stomach. ${ }^{(4)}$ This type of dysphagia has an incidence of $44 \%$ to $87 \% \%^{(3,5,6)}$ of cases after extubation, and it can increase morbidity and mortality. ${ }^{(7)}$ According to data collected from hospitals in the USA, the annual cost of patients with dysphagia exceeds US $\$ 500$ million. ${ }^{(4)}$

Recently, one of the concerns has been the study of dysphasia and its impact on the health care system. ${ }^{(8-11)}$ Dysphagia after prolonged OTI delays the resumption of oral feeding, increases the risk for lung diseases, and delays hospital discharge. ${ }^{(12,13)}$ In this scenario, early identification of predictors of aspiration is extremely relevant for priority treatment of at-risk patients, with the institution of appropriate measures and promotion of safe and speedy resumption of oral feeding. ${ }^{(14)}$ There are several tests available for the diagnosis of dysphagia after prolonged OTI, including bedside swallowing assessment, performed by a speech-language pathologist, as well as imaging (videofluoroscopy and videoendoscopy). ${ }^{(15)}$ However, it is of note that the use of such tests is not a reality in most treatment centers, in addition to the fact that indications for their use in critically ill patients are limited. ${ }^{(10)}$

A recent study ${ }^{(15)}$ investigated clinical predictors of dysphagia after prolonged OTI, on the basis of the results of clinical bedside swallowing assessments. The authors concluded that patients presenting with food or liquid escaping from the mouth or nose, multiple swallows, abnormal cervical auscultation findings, choking, altered voice quality, and cough after swallowing should be promptly evaluated before resumption of oral feeding. Other studies using bedside assessments have reported that the following signs are indicative of risk of aspiration: voice quality after swallowing ${ }^{(16,17)}$ and cough after swallowing. ${ }^{(17,18)}$

It is known that dysphagia severity after prolonged OTI is related to However, there are studies that have assessed the association of dysphagia with other risk factors, such as measures of patient severity of illness. ${ }^{(19-21)}$ In such studies, the authors included the results of instruments that determine patient severity of illness at the time of admission, such as the Sequential Organ Failure Assessment (SOFA) and the Acute Physiology and Chronic Health Evaluation II (APACHE II). ${ }^{(19-21)}$ 
Two current studies used SOFA scores calculated at ICU admission for investigating their correlations with dysphagia severity. ${ }^{(19,20)}$ Both studies found that patient severity of illness at the time of admission was not associated with dysphagia severity (determined by bedside assessment). Patients with mild dysphagia or normal swallowing had SOFA scores similar to those of patients with severe dysphagia.

Another study, ${ }^{(21)}$ the objective of which was to investigate the demographic and clinical factors associated with dysphagia after prolonged OTI in patients with pulmonary changes, analyzed the results of scales that measure severity of illness (SOFA and APACHE II scores) as possible factors related to post-extubation dysphagia severity. Results of the regression analysis showed that four variables were significant: duration of intubation; upper gastrointestinal comorbidity; SOFA score; and low BMI.

Therefore, the objective of the present study was to determine whether the severity of non-neurological critically ill patients correlates with clinical predictors of bronchial aspiration (bedside swallowing assessment).

\section{METHODS}

We conducted a prospective, observational, cross-sectional study, which was approved by the local research ethics committee (Code No. CAPPesq 311784). The data collection procedures began only after obtaining written informed consent from the patients or their legal guardians.

The study participants were patients who underwent bedside swallowing assessment, by physician request, and were being treated in the Department of Speech Therapy of the University of São Paulo School of Medicine Hospital das Clínicas Central Institute, located in the city of São Paulo, Brazil, between January of 2013 and January of 2015.

The inclusion criteria were having undergone prolonged OTI ( $>48 \mathrm{~h})^{(1-3)}$; being $>18$ years of age; not having or having had a tracheostomy; not having neurological or neurodegenerative disorders; not having a history of esophageal dysphagia; not having lung or neck cancer or having undergone surgical procedures in those regions; and having undergone bedside swallowing assessment within the first $48 \mathrm{~h}$ after extubation.

The steps in the data collection process of this study are described below.

\section{Clinical speech pathology assessment of the} risk of bronchial aspiration

The risk of bronchial aspiration was determined on the basis of the Dysphagia Risk Evaluation Protocol (DREP). ${ }^{(10,15)}$ The DREP is intended for early bedside assessment of the patient's risk of dysphagia. This protocol involves the controlled administration of water and puree volumes. The final result of the DREP determines whether the patient can receive larger volumes and different textures of foods and liquids, as well as indicating whether monitoring is required for safe feeding. The results observed for each protocol item are recorded as either "pass" or "fail".

A recently published study investigated predictors of dysphagia after prolonged OTI, ${ }^{(15)}$ on the basis of the DREP results for a $5-\mathrm{mL}$ water swallow test. In that study, the authors concluded that the predictors of dysphagia in the study population were food or liquid escaping from the mouth or nose, multiple swallows, abnormal cervical auscultation findings, voice quality after swallowing, cough after swallowing, and choking. Therefore, those DREP items were considered in the analysis and were assessed for correlations with the other data in the study.

\section{Level of swallowing function}

Level of swallowing function was determined with the use of the American Speech-Language-Hearing Association National Outcome Measurement System (ASHA NOMS) scale. (22) This scale is a multidimensional tool that assesses the degree of feeding supervision required by and the food/liquid textures that are safe for each patient, producing a single number between 1 and 7. For the purposes of the present study, the ASHA NOMS scale scores were determined after full completion of the DREP and, when necessary, after clinical swallowing assessment. Therefore, level of swallowing function was classified as follows: level 1: the individual is not able to swallow anything safely by mouth, and all nutrition and hydration are received through non-oral means; level 2: the individual is not able to swallow safely by mouth for nutrition and hydration, but may take some consistency, in therapy only, with consistent maximal cues, and an alternative method of feeding is required; level 3: an alternative method of feeding is required as the individual takes less than $50 \%$ of nutrition and hydration by mouth, and/or swallowing is safe with use of moderate cues to use compensatory strategies, and/or the individual requires maximum diet restriction; level 4: swallowing is safe, but usually requires moderate cues to use compensatory strategies, and/or the individual has moderate diet restrictions and/or still requires an alternative method of feeding and/or oral supplements; level 5: swallowing is safe with minimal diet restriction and/or occasionally requires minimal cues to use compensatory strategies, the individual may occasionally self-cue, and all nutrition and hydration needs are met by mouth at mealtime; level 6: swallowing is safe, and the individual eats and drinks independently, rarely requires minimal cues to use compensatory strategies, usually self-cues when difficulty occurs, but may need to avoid specific food items (e.g., popcorn and peanuts) and/or require additional time (because of dysphagia); and level 7: the individual's ability to eat independently is not limited by swallow function, which is safe and efficient for all consistencies, and compensatory strategies are effectively used when needed. 


\section{Patient severity of illness}

Patient severity of illness at the time of clinical speech pathology assessment was determined by using the SOFA score, ${ }^{(23)}$ recorded on the medical chart on the basis of clinical and laboratory test results. This score is a tool that is used on a daily basis in critically ill patients during their ICU stay, to determine the degree of organ dysfunction/failure quantitatively and objectively. The SOFA score is used not to determine patient outcome, but rather to describe complications in critically ill patients. The two major objectives of SOFA are to improve the understanding of organ dysfunction and how impairments in several organs are related to each other and to assess the effects of medical treatment.

To determine patient severity of illness, scores ranging from zero (normal) to four (highest degree of impairment) are assigned to the various organ systems (respiratory, cardiovascular, hematological, hepatic, central nervous system, and renal). Each organ system receives a separate score, and the final score is obtained by summing all scores. The maximum score is 20 , which is indicative of the highest degree of severity. The criteria for assigning points are described in Chart 1.

\section{Data analysis}

Assumptions of normality of distribution in each group and homogeneity of variances among the groups were tested using the Shapiro-Wilk test and Levene's test, respectively.

To compare SOFA scores among the different levels of swallowing function, we used the Kruskal-Wallis test, and, when multiple comparisons were necessary, we used Dunn's test. Categorical variables were analyzed with the chi-square test and Fisher's exact test. The level of statistical significance was set at 5\% ( $p \leq$ 0.05) for all analyses.

\section{RESULTS}

After the inclusion criteria were applied, the final study sample consisted of 150 patients. The underlying diagnoses of the patients included in the study were as follows: lung disease, in 59 patients; multiple trauma without traumatic brain injury, in 18; kidney and liver transplants, in 12; heart disease, in 11; vascular disease, in 11; liver disease, in 10; kidney disease, in 10; infectious disease, in 6 ; gastroenterological disease, in 5; rheumatic disease, in 5; and endocrine disease, in 3.

The distribution of the patients by level of swallowing function, as determined by the ASHA NOMS scale after bedside speech pathology assessment, was as follows: 3 patients at level $1 ; 35$ at level $2 ; 22$ at level 3; 27 at level $4 ; 12$ at level 5; 10 at level 6; and 41 at level 7. For the purposes of statistical analysis, the patients were grouped as follows: those at levels 1 and 2, ASHA1; those at levels 3 to 5, ASHA2; and those at levels 6 and 7, ASHA3. The group comparison by age and duration of intubation is shown in Table 1.

Statistical analysis using the Kruskal-Wallis test showed significant differences in age between the ASHA1 and ASHA3 groups ( $p<0.001)$ and between the ASHA2 and ASHA3 groups ( $p=0.026)$, patients in the ASHA3 group being younger than those in the other two groups. Mean duration of intubation (in days) was shortest in the ASHA3 group, and there was a significant difference between that group and the ASHA1 group ( $p=0.001)$.

Table 2 shows the comparison of predictors of bronchial aspiration among different levels of swallowing function.

Chart 1. Sequential Organ Failure Assessment scoring system.

\begin{tabular}{|c|c|c|c|c|c|}
\hline \multirow[t]{2}{*}{ Variable } & \multicolumn{5}{|c|}{ Score } \\
\hline & 0 & 1 & 2 & 3 & 4 \\
\hline \multicolumn{6}{|c|}{ Respiratory system } \\
\hline $\mathrm{PaO}_{2} / \mathrm{FiO}_{2}, \mathrm{mmHg}$ & $>400$ & $\leq 400$ & $\leq 300$ & $\leq 200^{a}$ & $\leq 100^{\mathrm{a}}$ \\
\hline \multicolumn{6}{|c|}{ Hematological system } \\
\hline Platelets, $\times 10^{3} / \mathrm{mm}^{3}$ & $>150$ & $\leq 150$ & $\leq 100$ & $\leq 50$ & $\leq 20$ \\
\hline \multicolumn{6}{|c|}{ Hepatic system } \\
\hline Bilirubin, mg/dL ( $\mu \mathrm{mol} / \mathrm{L})$ & $<1.2(<20)$ & $1.2-1.9(20-32)$ & $2.0-5.9(33-101)$ & $6.0-11.9(102-204)$ & $>12.0(>204)$ \\
\hline \multicolumn{6}{|c|}{ Cardiovascular system } \\
\hline Hypotension & No hypotension & $\mathrm{MAP}<70 \mathrm{mmHg}$ & 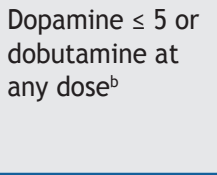 & $\begin{array}{l}\text { Dopamine }>5 \text {, } \\
\text { epinephrine } \leq 1 \text {, } \\
\text { or norepinephrine } \\
\leq 0.1^{\mathrm{b}}\end{array}$ & $\begin{array}{l}\text { Dopamine }>15 \text {, } \\
\text { epinephrine } \\
>0.1 \text {, or } \\
\text { norepinephrine } \\
>0.1^{b}\end{array}$ \\
\hline \multicolumn{6}{|c|}{ CNS } \\
\hline Glasgow Coma Scale & 15 & $13-14$ & $10-12$ & $6-9$ & $<6$ \\
\hline \multicolumn{6}{|c|}{ Renal system } \\
\hline Creatinine, mg/dL ( $\mu \mathrm{mol} / \mathrm{L})$ & $<1.2(<110)$ & $1.2-1.9(110-170)$ & $2.0-3.4(171-299)$ & $3.5-4.9(300-440)$ & $>5.0(>440)$ \\
\hline Urine output, $\mathrm{mL} /$ day & $\mathrm{N} / \mathrm{A}$ & N/A & $\mathrm{N} / \mathrm{A}$ & $<500$ & $<200$ \\
\hline
\end{tabular}

MAP: mean arterial pressure; and SNC central nervous system. ${ }^{\text {aW }}$ ith ventilatory support. ${ }^{\mathrm{b}}$ Adrenergic agents $(\mu \mathrm{g} /$ $\mathrm{kg}$ body weight/min) were administered for at least $1 \mathrm{~h}$. 
Table 1. Age and duration of intubation in the patients studied.

\begin{tabular}{lcccc}
\multicolumn{1}{c}{ Variable } & Group & p* \\
& ASHA1 & ASHA2 & ASHA3 & $<0.001$ \\
Age, years & $62.00 \pm 17.40$ & $55.30 \pm 17.48$ & $46.40 \pm 18.30$ & $<0.001$ \\
Duration of intubation, days & $7.60 \pm 3.97$ & $6.20 \pm 3.38$ & $4.90 \pm 2.70$ & $<$ \\
\hline
\end{tabular}

ASHA1: patients at levels 1 and 2, as scored by the American Speech-Language-Hearing Association National Outcome Measurement System (ASHA NOMS); ASHA2: patients at levels 3, 4, and 5, as scored by ASHA NOMS; and ASHA3: patients at levels 6 and 7, as scored by ASHA NOMS. *ANOVA.

Table 2. Comparison of predictors of bronchial aspiration among levels of swallowing function.

\begin{tabular}{|c|c|c|c|c|c|}
\hline \multirow[t]{2}{*}{ Clinical sign } & \multirow[t]{2}{*}{ Result } & \multicolumn{3}{|c|}{ Group } & \multirow[t]{2}{*}{ p* } \\
\hline & & $\begin{array}{c}\text { ASHA1 } \\
(n=38)\end{array}$ & $\begin{array}{c}\text { ASHA2 } \\
(n=61)\end{array}$ & $\begin{array}{c}\text { ASHA3 } \\
(n=51)\end{array}$ & \\
\hline \multirow[t]{2}{*}{ Extraoral loss ${ }^{\mathrm{a}}$} & Pass & 35 & 57 & 50 & 0.402 \\
\hline & Fail & 3 & 4 & 1 & \\
\hline \multirow[t]{2}{*}{ Multiple swallows } & Pass & 24 & 42 & 44 & 0.030 \\
\hline & Fail & 14 & 19 & 7 & \\
\hline \multirow[t]{2}{*}{ Abnormal cervical auscultation findings } & Pass & 21 & 52 & 51 & $<0.001$ \\
\hline & Fail & 17 & 9 & 0 & \\
\hline \multirow[t]{2}{*}{ Wet voice } & Pass & 31 & 55 & 51 & 0.009 \\
\hline & Fail & 7 & 6 & 0 & \\
\hline \multirow[t]{2}{*}{ Cough } & Pass & 16 & 37 & 50 & $<0.001$ \\
\hline & Fail & 22 & 24 & 1 & \\
\hline \multirow[t]{2}{*}{ Choking } & Pass & 31 & 52 & 51 & 0.008 \\
\hline & Fail & 7 & 9 & 0 & \\
\hline
\end{tabular}

ASHA1: patients at levels 1 and 2, as scored by the American Speech-Language-Hearing Association National Outcome Measurement System (ASHA NOMS); ASHA2: patients at levels 3, 4, and 5, as scored by ASHA NOMS; and ASHA3: patients at levels 6 and 7, as scored by ASHA NOMS. aFood or liquid escaping from the mouth or nose. *Chi-square test.

The results indicated that the predictor "food or liquid escaping from the mouth or nose" did not differentiate among the swallowing function level groups. The predictors "abnormal cervical auscultation findings" and "cough" after swallowing were the indicators that best differentiated among the groups. To determine the groups for which the predictors of aspiration were the most evident, we used Fisher's exact test. In that analysis, the groups were matched for predictors of aspiration-multiple swallows: ASHA1 vs. ASHA2 ( $p$ $=0.559)$; ASHA1 vs. ASHA3 ( $p=0.011)$; and ASHA2 vs. ASHA3 ( $p=0.030)$; abnormal cervical auscultation findings: ASHA1 vs. ASHA2 ( $p=0.001) ;$ ASHA1 vs. ASHA3 ( $p<0.001)$; and ASHA2 vs. ASHA3 ( $p=0.004)$; wet voice: ASHA1 vs. ASHA2 ( $p=0.236)$; ASHA1 vs. ASHA3 ( $p=0.002)$; and ASHA2 vs. ASHA3 ( $p=$ $0.031)$; cough after swallowing: ASHA1 vs. ASHA2 ( $p$ $=0.072)$; ASHA1 vs. ASHA3 ( $p<0.001)$; and ASHA2 vs. ASHA3 ( $p<0.001)$; and choking: ASHA1 vs. ASHA2 ( $p=0.630)$; ASHA1 vs. ASHA3 ( $p=0.002)$; and ASHA2 vs. ASHA3 ( $p=0.004)$.

Tables 3 and 4 show the results of the comparison of patient severity of illness (SOFA) among levels of swallowing function at the time of speech pathology assessment.

The results of the two analyses indicate that poor swallowing function translates to greater patient severity of illness. It can also be seen that the ASHA1 group (patients who cannot tolerate oral feeding) and the ASHA2 group (patients who require maximum to moderate diet restriction) did not differ regarding patient severity of illness (SOFA score).

To establish which indicators of patient severity of illness could have an impact on swallowing, we sought to determine whether indicators of functioning of the respiratory, cardiovascular, and central nervous systems (SOFA score) correlate with level of swallowing function at the time of speech pathology assessment (Table 5). The results indicated that only changes in the central nervous system correlated with changes in swallowing function, indicating that poor swallowing function translates to central nervous system impairment. In an analysis solely of the indicator of functioning of the central nervous system, Dunn's test indicated that the group with the poorest swallowing function differed from the others: ASHA1 vs. ASHA2 ( $p=$ $0.018)$; ASHA1 vs. ASHA3 ( $p<0.001)$; and ASHA2 vs. ASHA3 ( $p=0.101)$.

\section{DISCUSSION}

This study presents the characteristics of the risk of bronchial aspiration in the largest sample of patients after prolonged OTI in Brazil. Establishing priority indicators for post-extubation speech pathology care is essential to reducing hospital costs, optimizing bedside speech pathology assessment, and promoting safe and speedy resumption of oral feeding.

The literature indicates that the impact of age on the occurrence of dysphagia after prolonged OTI is 
Table 3. Comparison of patient severity of illness among levels of swallowing function.

\begin{tabular}{cccc}
$\begin{array}{c}\text { Level of } \\
\text { wallowing } \\
\text { function }\end{array}$ & Median & IQR & p \\
\hline ASHA1 & 5.0 & $3.75-8.00$ & 0.001 \\
ASHA2 & 5.0 & $3.00-7.00$ & \\
ASHA3 & 3.0 & $2.00-5.00$ & \\
\hline
\end{tabular}

IQR: interquartile range; ASHA1: patients at levels 1 and 2, as scored by the American Speech-LanguageHearing Association National Outcome Measurement System (ASHA NOMS); ASHA2: patients at levels 3, 4, and 5, as scored by ASHA NOMS; and ASHA3: patients at levels 6 and 7, as scored by ASHA NOMS. *KruskalWallis test.

similar in young adults and in the elderly(24,25); both groups seem to differ only in dysphagia resolution, the elderly having a trend toward delayed resumption of oral feeding. ${ }^{(2,18,26)}$ In contrast, the present study showed that predictors of bronchial aspiration were more common in individuals 55 years of age or older.

A previous study reported the impact of aging on the swallowing function, indicating that healthy elderly individuals present with vallecular residuals (laryngeal retention) and that such individuals are usually unaware of retained food in the pharynx. ${ }^{(27)}$ Although pharyngeal clearing is nearly complete in young, asymptomatic individuals, the same is not true for healthy elderly individuals. (27) The mechanisms responsible for the development of pharyngeal retention have yet to be fully determined. According to the literature, ${ }^{(28,29)}$ age-related physiological changes-reduced amplitude of pharyngeal contractions, pharyngeal shortening, reduced tongue propulsion/strength, and reduced soft palate strength, all of which hinder the displacement of the food bolus-may be involved in the impairment of the swallowing process. Those factors could also explain the higher frequency of occurrence of cough and abnormal auscultation findings after swallowing, observed in the present study, in the critically ill patients 55 years of age or older.

The association between duration of orotracheal intubation and dysphagia severity is well documented

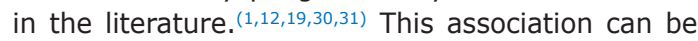
explained by the impact of the tube remaining in the oral cavity, pharynx, and larynx. It is known that chemoreceptors and/or mechanoreceptors, located in the pharyngeal and laryngeal mucosa and involved in the swallowing reflex, can undergo changes due to the presence of an orotracheal tube. ${ }^{(3)}$ Laryngeal sensory impairment, identified by the absence of cough or of any other clinical sign suggestive of aspiration, has been observed in patients during the ingestion of liquids, immediately after extubation and within $4 \mathrm{~h}$ after extubation. ${ }^{(32,33)}$ The results of the present study suggest that predictors of aspiration are more common in individuals who remain intubated for 6 days or more.

The present study identified abnormal cervical auscultation findings as a possible predictor of bronchial aspiration. The sounds associated with healthy and
Table 4. Multiple comparisons of patient severity of illness among levels of swallowing function.

\begin{tabular}{cc} 
Comparison & $\mathbf{p}$ \\
ASHA1 vs. ASHA2 & $>0.999$ \\
ASHA1 vs. ASHA3 & 0.003 \\
ASHA2 vs. ASHA3 & 0.005 \\
\hline
\end{tabular}

ASHA1: patients at levels 1 and 2, as scored by the American Speech-Language-Hearing Association National Outcome Measurement System (ASHA NOMS); ASHA2: patients at levels 3, 4, and 5, as scored by ASHA NOMS; and ASHA3: patients at levels 6 and 7, as scored by ASHA NOMS.*Dunn's test.

Table 5. Comparison of the severity of involvement of the respiratory, cardiovascular, and central nervous systems among levels of swallowing function.

\begin{tabular}{ccccc}
$\begin{array}{c}\text { SOFA } \\
\text { score }\end{array}$ & $\begin{array}{c}\text { ASHA1 } \\
(\mathbf{n}=\mathbf{3 8})\end{array}$ & $\begin{array}{c}\text { ASHA2 } \\
\text { (n = 61) }\end{array}$ & $\begin{array}{c}\text { ASHA3 } \\
(\mathbf{n}=\mathbf{5 1})\end{array}$ \\
\hline 0 & 8 & 13 & 14 & 0.436 \\
1 & 14 & 13 & 15 & \\
2 & 9 & 18 & 7 & \\
3 & 7 & 14 & 13 & \\
4 & 0 & 3 & 2 & \\
\hline \multicolumn{5}{c}{ Respiratory } \\
\hline 0 & 28 & 49 & 47 & 0.507 \\
1 & 2 & 3 & 1 & \\
2 & 1 & 1 & 1 & \\
3 & 5 & 7 & 1 & \\
4 & 2 & 1 & 1 & \\
\hline 0 & 15 & 40 & 43 & 0.001 \\
1 & 18 & 18 & 8 & \\
\hline 3 & 4 & 2 & 0 & \\
4 & 3 & 1 & 0 & \\
\hline
\end{tabular}

SOFA: Sequential Organ Failure Assessment; ASHA1: patients at levels 1 and 2, as scored by the American Speech-Language-Hearing Association National Outcome Measurement System (ASHA NOMS); ASHA2: patients at levels 3,4 , and 5 , as scored by ASHA NOMS; ASHA3: patients at levels 6 and 7, as scored by ASHA NOMS; and CNS: central nervous system. ${ }^{*}$ Chi-square test.

pathological swallowing have been identified with the aid of accelerometers and microphones, for analysis of acoustic characteristics ${ }^{(34,35)}$ and determination of the characteristic sounds of swallowing. ${ }^{(35-37)}$ The results regarding the accuracy of the method vary considerably in terms of reliability and validity in comparison with those of imaging. ${ }^{(38)}$ In contrast, it should be taken into consideration that the very interpretation of videofluoroscopy images and results also varies in terms of reliability and reproducibility. $(39,40)$ Regardless of the method adopted to assess swallowing, there is a consensus that specific training in its use is indispensable.

Data analysis indicated that, in the present study, critically ill patients who underwent prolonged OTI and had poorer swallowing function had greater overall 
severity of illness, changes in the central nervous system being the factor with the greatest impact on this difference. As shown in the introduction, the results of the studies that correlated patient severity of illness (SOFA score) with dysphagia severity did not achieve significance. ${ }^{(19,20)}$ However, the item regarding neurological system impairment was not included in the analyses of those studies. The literature has identified that a decreased level of consciousness has impacts on the swallowing mechanism and can lead to aspiration. $(2,5,31)$

Finally, the limitations of the present study should be acknowledged. First, the study sample consisted of patients from a single institution, and, therefore, the results might have some bias resulting from the therapeutic approaches adopted in the specific protocols. Second, the type of assessment proposed for identifying predictors of bronchial aspiration was based solely on an observational clinical protocol. Determination of the actual occurrence of bronchial aspiration, on the basis of the signs evaluated, was not objectively confirmed by imaging. It is of note that the present study did not exclude full speech pathology assessments, including imaging.

The results of the present study suggest that critically ill patients 55 years of age or older who undergo prolonged OTI ( $\geq 6$ days), have a SOFA score $\geq 5$, have a Glasgow Coma Scale score $\leq 14$, and present with abnormal cervical auscultation findings or cough after swallowing (5 $\mathrm{mL}$ water swallow test at the bedside) should be prioritized for a full speech pathology assessment and, if necessary, referred for imaging confirmation.

\section{REFERENCES}

1. Brown CV, Hejl K, Mandaville AD, Chaney PE, Stevenson G, Smith C. Swallowing dysfunction after mechanical ventilation in trauma patients. J Crit Care. 2011;26(1):108.e9-13. http://dx.doi. org/10.1016/j.jcrc.2010.05.036

2. Barquist E, Brown M, Cohn S, Lundy D, Jackowski J. Postextubation fiberoptic endoscopic evaluation of swallowing after prolonged endotracheal intubation: a randomized, prospective trial. Crit Care Med. 2001;29(9):1710-3. http://dx.doi.org/10.1097/00003246200109000-00009

3. de Larminat V, Montravers P, Dureuil B, Desmonts JM. Alteration in swallowing reflex after extubation in intensive care unit patients. Crit Care Med. 1995;23(3):486-90. http://dx.doi.org/10.1097/00003246199503000-00012

4. Macht, M, Wimbish T, Clark BJ, Benson AB, Burnham EL, Williams $A$, et al. Diagnosis and treatment of post-extubation dysphagia: results from a national survey. J Crit Care. 2012;27(6):578-86. http:// dx.doi.org/10.1016/j.jcrc.2012.07.016

5. Skoretz SA, Flowers HL, Martino R. The incidence of dysphagia following endotracheal intubation: a systematic review. Chest. 2010;137(3):665-73. http://dx.doi.org/10.1378/chest.09-1823

6. Elpern EH, Scott MG, Petro L, Ries MH. Pulmonary aspiration in mechanically ventilated patients with tracheostomies. Chest. 1994;105(2):563-6. http://dx.doi.org/10.1378/chest.105.2.563

7. Tolep K, Getch CL, Criner GJ. Swallowing dysfunction in patients receiving prolonged mechanical ventilation. Chest. 1996;109(1):16772. http://dx.doi.org/10.1378/chest.109.1.167

8. Altman KW, Yu GP, Schaefer SD. Consequence of dysphagia in the hospitalized patient: impact on prognosis and hospital resources. Arch Otolaryngol Head Neck Surg. 2010;136(8):784-9. http://dx.doi. org/10.1001/archoto.2010.129

9. Brazilian recommendations of mechanical ventilation 2013. Part 2 J Bras Pneumol. 2014;40(5):458-86. http://dx.doi.org/10.1590/S180637132014000500003

10. Moraes, DP, Sassi FC, Mangilli LD, Zilberstein B, de Andrade CR Clinical prognostic indicators of dysphagia following prolonged orotracheal intubation in ICU patients. Crit Care. 2013;17(5):R243. http://dx.doi.org/10.1186/cc13069

11. Hu X, Yi ES, Ryu JH. Diffuse aspiration bronchiolitis: analysis of 20 consecutive patients. J Bras Pneumol. 2015;41(2):161-6. http:// dx.doi.org/10.1590/S1806-37132015000004516

12. Barker J, Martino R, Reichardt B, Hickey EJ, Ralph-Edwards A. Incidence and impact of dysphagia in patients receiving prolonged endotracheal intubation after cardiac surgery. Can J Surg. 2009;52(2):119-24

13. Ward EC, Green K, Morton AL. Patterns and predictors of swallowing resolution following adult traumatic brain injury. J Head Trauma Rehabil. 2007;22(3):184-91. http://dx.doi.org/10.1097/01. HTR.0000271119.96780.f5

14. Altman KW, Yu GP, Schaefer SD. Consequence of dysphagia in the hospitalized patient: impact on prognosis and hospital resources. Arch Otolaryngol Head Neck Surg. 2010;136(8):784-9. http://dx.doi. org/10.1001/archoto.2010.129
15. Medeiros GC, Sassi FC, Mangilli LD, Zilberstein B, Andrade CR. Clinical dysphagia risk predictors after prolonged orotracheal intubation. Clinics (Sao Paulo). 2014;69(1):8-14. http://dx.doi. org/10.6061/clinics/2014(01)02

16. Martino R, Silver F, Teasell R, Bayley M, Nicholson G, Streiner DL, et al. The Toronto Bedside Swallowing Screening Test (TOR-BSST): development and validation of a dysphagia screening tool for patients with stroke. Stroke. 2009;40(2):555-61. http://dx.doi.org/10.1161/ STROKEAHA.107.510370

17. Edmiaston J, Connor LT, Steger-May K, Ford AL. A simple bedside stroke dysphagia screen, validated against videofluoroscopy, detects dysphagia and aspiration with high sensitivity. J Stroke Cerebrovasc Dis. 2014;23(4):712-6. http://dx.doi.org/10.1016/j. jstrokecerebrovasdis.2013.06.030

18. Bordon A, Bokhari R, Sperry J, Testa D 4th, Feinstein A, Ghaemmaghami V. Swallowing dysfunction after prolonged intubation: analysis of risk factors in trauma patients. Am J Surg. 2011;202(6):679-82. http://dx.doi.org/10.1016/j.amjsurg.2011.06.030

19. Macht M, Wimbish T, Clark BJ, Benson AB, Burnham EL, Williams A, et al. Postextubation dysphagia is persistent and associated with poor outcomes in survivors of critical illness. Crit Care. 2011;15(5):R231. http://dx.doi.org/10.1186/cc10472

20. Macht M, King CJ, Wimbish T, Clark BJ, Benson AB, Burnham EL, et al. Post-extubation dysphagia is associated with longer hospitalization in survivors of critical illness with neurologic impairment. Crit Care. 2013;17(3):R119. http://dx.doi.org/10.1186/cc12791

21. Brodsky, BM, Gellar JE, Dinglas VD, Colantuoni E, Mendez-Tellez PA, Shanholtz $C$, et al. Duration of oral endotracheal intubation is associated with dysphagia symptoms in acute lung injury patients. J Crit Care. 2014;29(4):574-9. http://dx.doi.org/10.1016/j. jcrc.2014.02.015

22. American Speech-Language-Hearing Association; National Outcome Measurement System (NOMS). Adult Speech-Language Pathology Training Manual. Rockville, MD: American Speech-Language-Hearing Association; 1998.

23. Moreno R, Vincent JL, Matos R, Mendonça A, Cantraine F, Thijs L, et al. The use of maximum SOFA score to quantify organ dysfunction/ failure in intensive care. Results of a prospective, multicenter study. Working Group on Sepsis related Problems of the ESICM. Intensive Care Med. 1999;25(7):686-96. http://dx.doi.org/10.1007/ s001340050931

24. McMicken BL, Muzzy CL. Prognostic indicators of functional outcomes in first time documented acute stroke patients following standard dysphagia treatment. Disabil Rehabil. 2009;31(26): 2196203. http://dx.doi.org/10.3109/09638280902956894

25. Schroeder MF, Daniels SK, McClain M, Corey DM, Foundas AL. Clinical and cognitive predictors of swallowing recovery in stroke. J Rehabil Res Dev. 2006;43(3):301-10. http://dx.doi.org/10.1682/ JRRD.2004.12.0154

26. Leder SB, Suiter DM. An epidemiologic study on aging and dysphagia in the acute care hospitalized population: 2000-2007. Gerontology. 2009;55(6):714-8. http://dx.doi.org/10.1159/000235824

27. Yoshikawa M, Yoshida M, Nagasaki T, Tanimoto K, Tsuga K, Akagawa 
$Y$, et al. Aspects of swallowing in healthy dentate elderly persons older than 80 years. J Gerontol A Biol Med Sci. 2005;60(4):506-9. http://dx.doi.org/10.1093/gerona/60.4.506

28. Kallman DA, Plato CC, Tobin JD. The role of muscle loss in the agerelated decline of grip strength: cross-sectional and longitudina perspectives. J Gerontol. 1990;45(3):M82-8. http://dx.doi. org/10.1093/geronj/45.3.M82

29. Dejaeger $E$, Pelemans W, Ponette $E$, Joosten E. Mechanisms involved in postdeglutition retention in elderly. Dysphagia. 1997;12(2):63-7. http://dx.doi.org/10.1007/PL00009520

30. Skoretz SA, Yau TM, Ivanov J, Granton JT, Martino R. Dysphagia and associated risk factors following extubation in cardiovascular surgical patients. Dysphagia. 2014;29(6):647-54. http://dx.doi.org/10.1007/ s00455-014-9555-4

31. Kwok AM, Davis JW, Cagle KM, Sue LP, Kaups KL. Post-extubation dysphagia in trauma patients: it's hard to swallow. Am J Surg. 2013;206(6):924-7; discussion 927-8. http://dx.doi.org/10.1016/]. amjsurg.2013.08.010

32. Burgess GE 3rd, Cooper JR Jr, Marino RJ, Peuler MJ, Warriner RA 3rd. Laryngeal competence after tracheal extubation. Anesthesiology. 1979;51(1):73-7. http://dx.doi.org/10.1097/00000542-197907000 00016

33. Stauffer JL, Olson DE, Petty TL. Complications and consequences of endotracheal intubation and tracheotomy. A prospective study of 150 critically ill adult patients. Am J Med. 1981;70(1):65-76. http://dx.doi. org/10.1016/0002-9343(81)90413-7

34. Morinièri $S$, Beutter $P$, Boiron $M$. Sound component duration of healthy human pharyngoesophageal swallowing: a gender comparison study. Dysphagia. 2006:21(3):175-82. http://dx.doi. org/10.1007/s00455-006-9023-x

35. Cichero JA, Murdoch BE. Acoustic signature of the normal swallow: characterization by age, gender, and bolus volume Ann Otol Rhinol Laryngol. 2002;111(7 Pt 1):623-32. http://dx.doi. org/10.1177/000348940211100710

36. Zenner PM, Losinski DS, Millis RH. Using cervical auscultation in the clinical dysphagia examination in long-term care. Dysphagia. 1995;10(1):27-31. http://dx.doi.org/10.1007/BF00261276

37. Lazareck LJ, Moussavi ZM. Classification of normal and dysphagic swallows by acoustical means. IEEE Trans Biomed Eng 2004;51(12):2103-12. http://dx.doi.org/10.1109/TBME.2004.836504

38. Mann G, Hankey GJ. Initial clinical and demographic predictors of swallowing impairment following acute stroke. Dysphagia. 2001;16(3):208-15. http://dx.doi.org/10.1007/s00455-001-0069-5

39. Kuhlemeier KV, Yates P, Palmer JB. Intra- and interrater variation in the evaluation of videofluorographic swallowing studies. Dysphagia 1998;13(3):142-7. http://dx.doi.org/10.1007/PL00009564

40. McCullough GH, Wertz RT, Rosenbek JC, Mills RH, Webb WG, Ross $\mathrm{KB}$. Inter- and intrajudge reliability for videofluoroscopic swallowing evaluation measures. Dysphagia. 2001;16(2):110-8. http://dx.doi. org/10.1007/PL00021291 